Z Epileptol 2012 $25: 21-21$

DOI 10.1007/s10309-011-0180-z

Online publiziert: 3. Februar 2012

(c) Springer-Verlag 2012

\title{
H. Potschka
}

Institut für Pharmakologie, Toxikologie und Pharmazie, Ludwig-Maximilians-Universität, München

\section{Alfred-Hauptmann-Preis}

Der nach dem deutschen Neurologen und Psychiater Alfred Hauptmann benannte Preis wird für die beste wissenschaftliche Arbeit aus dem deutschsprachigen Raum auf dem Gebiet der experimentellen und klinischen Epileptologie vergeben. Wie in der letzten Ausgabe der Zeitschrift für Epileptologie bereits berichtet, wurde der Alfred-Hauptmann-Preis dieses Jahr an Frau Claudia Brandt, PhD, und Herrn Prof. Ingmar Blümcke verliehen.

Frau Claudia Brandt, $P h D$, ist wissenschaftliche Mitarbeiterin in der Arbeitsgruppe von Herrn Prof. Wolfgang Löscher am Institut für Pharmakologie, Toxikologie und Pharmazie der Stiftung Tierärztliche Hochschule Hannover. Sie beschäftigt sich seit langen Jahren engagiert mit der Entwicklung und Prüfung verschiedener Strategien zur Therapie und Prävention von Epilepsien. Die zahlreichen Forschungsprojekte mündeten in einer Vielzahl von Publikationen, die zum heutigen Kenntnisstand hinsichtlich der Epilepsieentstehung und der Resistenz von Epilepsien wesentlich beitragen.

In der Arbeit, die durch den AlfredHauptmann-Preis ausgezeichnet wurde, untersuchten Claudia Brandt und Kollegen, ob die Entstehung von Epilepsien durch eine indirekte pharmakologische Modulation der GABA $_{\mathrm{A}}$-Rezeptor vermittelten Ionenströme verhindert werden kann [1].

In dieser Ausgabe der Zeitschrift für Epileptologie gibt Claudia Brandt eine Übersicht über den aktuellen Kenntnisstand zu Veränderungen der $\mathrm{GABA}_{\mathrm{A}}$-Rezeptor-Funktion im epileptischen Gehirn und fasst die wesentlichen Erkenntnisse aus der ausgezeichneten Forschungsarbeit zusammen (s. S. 22 ff).

Hinsichtlich der zweiten ausgezeichneten Arbeit verweisen wir auf die letzte Ausgabe, in der wir den Preisträger Prof.
Ingmar Blümcke näher vorgestellt haben und in der Ingmar Blümcke über seine Forschungsarbeit berichtete.

Heidrun Potschka, München

\section{Korrespondenzadresse}

\section{Prof. Dr. H. Potschka}

Institut für Pharmakologie, Toxikologie und Pharmazie, Ludwig-Maximilians-Universität Koeniginstr. 16, 80539 München

potschka@pharmtox.vetmed.uni-muenchen.de

Interessenkonflikt. Die korrespondierende Autorin gibt an, dass kein Interessenkonflikt besteht.

\section{Literatur}

1. Brandt C, Nozadze M, Heuchert N et al (2010) Disease-modifying effects of phenobarbital and the NKCC1 inhibitor bumetanide in the pilocarpine model of temporal lobe epilepsy. J Neurosci 30:8602-8612 